\title{
Rational Chebyshev functions with new collocation points in semi infinite domains for solving higher-order linear ordinary differential equations
}

Mohamed A. Ramadan*a, Kamal. R. Raslan**

Adel R. Hadhoud ${ }^{* b}$ and Mahmoud A. Nassar **

${ }^{*}$ Mathematics Department, Faculty of Science, Menoufia University, Shebein El-Koom, Egypt.

${ }^{* b}$ M.Sc. \& Ph.D. from Mathematics Department, Faculty of Science, Menoufia University, Shebein El-Koom, Egypt

${ }^{* *}$ Mathematics Department, Faculty of Science, Al-Azhar University, Nasr-City, Cairo, Egypt.

\section{ABSTRACT}

The purpose of this paper is to investigate the use of rational Chebyshev $(R C)$ functions for solving higher-order linear ordinary differential equations with variable coefficients on a semi-infinite domain using new rational Chebyshev collocation points. This method transforms the higher-order linear ordinary differential equations and the given conditions to matrix equations with unknown rational Chebyshev coefficients. These matrices together with the collocation method are utilized to reduce the solution of higher-order ordinary differential equations to the solution of a system of algebraic equations. The solution is obtained in terms of RC series. Numerical examples are given to demonstrate the validity and applicability of the method. The obtained numerical results are compared with others existing methods and the exact solution where it shown to be very attractive and maintains better accuracy.

Keywords: Rational Chebyshev functions; higher-order ordinary differential equations;rational Chebyshev collocation method.

MSC: 26A33; 65L05; 65D15

\section{Council for Innovative Research}

Peer Review Research Publishing System

Journal: JOURNAL OF ADVANCES IN MATHEMATICS

Vol .11, No.7

www.cirjam.com , editorjam@gmail.com 


\section{1- INTRODUCTION}

The well-known Chebyshev polynomial of first kind $T_{n}(x)[1]$ are orthogonal with respect to the weight-function $w(x)=1 / \sqrt{1-x^{2}}$ on the interval $[-1,1]$, and the recurrence relation is

$$
T_{0}(x)=1, \quad T_{1}(x)=x, \quad T_{n+1}(x)=2 x T_{n}(x)-T_{n-1}(x) \quad n \geq 1
$$

These polynomials have many applications in numerical analysis, and a lot of studies are devoted to show the merits of them in various ways. One of the applications of Chebyshev polynomials is the solution of ordinary differential equations with boundary conditions [1, 2]. Many studies are considered on the interval $[-1,1]$ in which Chebyshev polynomials are defined. Therefore, this limitation causes a failure of the Chebyshev approach in the problems that are naturally defined on larger domains, especially including infinity. Under a transformation that maps the interval $[-1,1]$ into a semi-infinite domain $[0, \infty)$, Boyd [3, 4]. Also Parand et al. [5, 6], Sezer et al. [7, 8] and Ramadan et al. [9, 10] successfully applied spectral methods to solve problems on open semi-infinite intervals. In their studies, the basis functions called rational Chebyshev functions $R_{n}(x)$ and defined by

$$
R_{n}(x)=T_{n}\left(\frac{x-1}{x+1}\right) \quad x \in[0, \infty)
$$

The advantage of this method is applied spectral method to solve problems on semi-infinite intervals and deals directly with infinite boundary without singularities.

\section{3- Properties of the rational Chebyshev (RC) functions}

The rational Chebyshev functions are orthogonal in the interval $[0, \infty)$, with respect to the weight function $w(t)=1 /((t+1) \sqrt{t})$ and can be determined with the aid of the recurrence formulae

$$
R_{0}(x)=1, \quad R_{l}(x)=\frac{x-1}{x+1}, \quad R_{n+1}=2\left(\frac{x-1}{x+1}\right) R_{n}(x)-R_{n-1}(x) \quad n \geq 1
$$

If we use the expression $v=\frac{x-1}{x+1}$ in the $\mathrm{RC}$ function (2), we have

$$
R(x)=V(x) C^{T}
$$

where $R(x)$ and $V(x)$ are matrices of the form:

$$
\begin{aligned}
& R(x)=\left[\begin{array}{llll}
R_{0}(x) & R_{1}(x) & \ldots & R_{N}(x)
\end{array}\right] \\
& V(x)=\left[\begin{array}{llll}
v^{0}(x) & v^{1}(x) & \ldots & v^{N}(x)
\end{array}\right]
\end{aligned}
$$

and $C^{T}$ is a matrix with its inverse given by

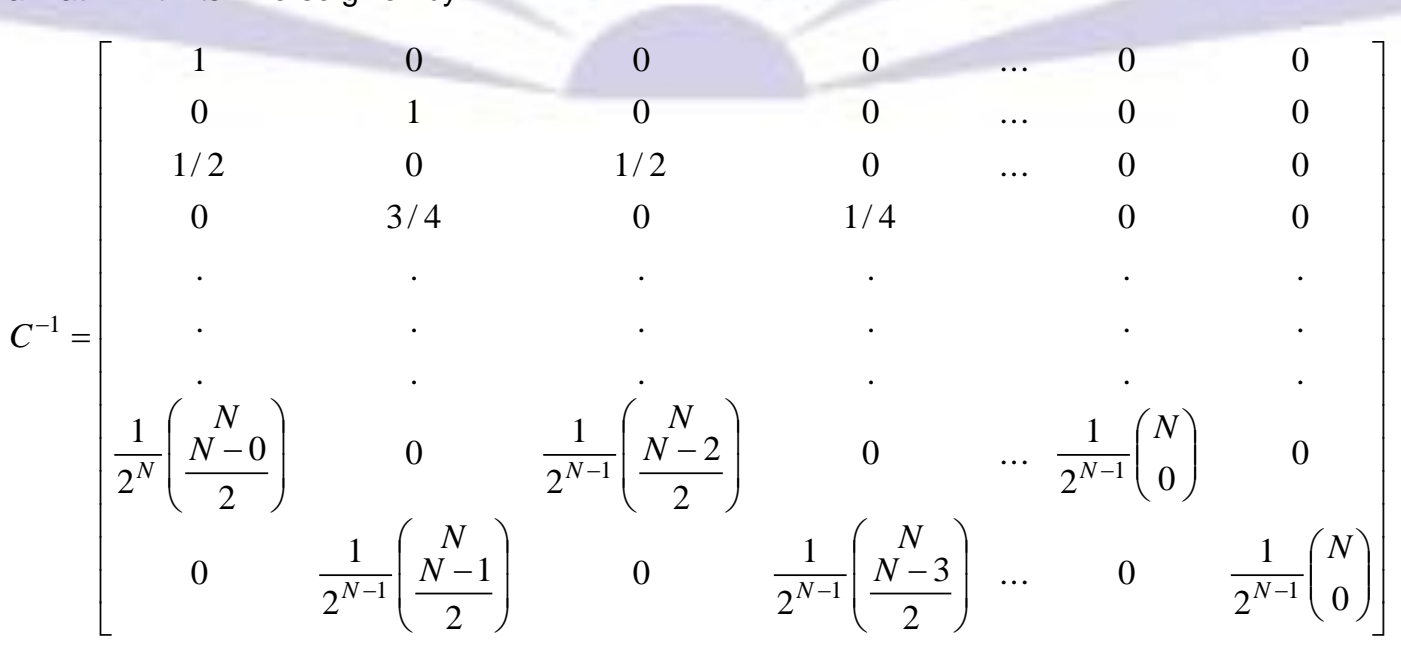

In this case, we are going to use the last row for odd values of $N$, and the second row from below of matrix $C^{-1}$. 
For example, in the cases $N=3$ and $N=4$, the matrix $C$ becomes

$$
C=\left[\begin{array}{cccc}
1 & 0 & 0 & 0 \\
0 & 1 & 0 & 0 \\
-1 & 0 & 2 & 0 \\
0 & -3 & 0 & 4
\end{array}\right], \quad C=\left[\begin{array}{ccccc}
1 & 0 & 0 & 0 & 0 \\
0 & 1 & 0 & 0 & 0 \\
-1 & 0 & 2 & 0 & 0 \\
0 & -3 & 0 & 4 & 0 \\
1 & 0 & -8 & 0 & 8
\end{array}\right]
$$

Consequently, the $j^{\text {th }}$ derivative of the matrix $R(x)$, can be obtained as

$$
R^{(j)}(x)=V^{(j)}(x) C^{T}
$$

where

$$
\begin{aligned}
& R^{(j)}(x)=\left[\begin{array}{llll}
R_{0}^{(j)}(x) & R_{1}^{(j)}(x) & \ldots & R_{N}^{(j)}(x)
\end{array}\right] \\
& V^{(j)}(x)=\left[\begin{array}{llll}
\left(v^{0}(x)\right)^{(j)} & \left(v^{1}(x)\right)^{(j)} & \ldots & \left(v^{N}(x)\right)^{(j)}
\end{array}\right]
\end{aligned}
$$

and

$$
v^{0}(x)=1, \quad v^{1}(x)=\frac{x-1}{x+1}, \quad v^{2}(x)=\left(\frac{x-1}{x+1}\right)^{2}, \quad \ldots, \quad v^{N}(x)=\left(\frac{x-1}{x+1}\right)^{N}
$$

\section{Problem statement}

The form of higher-order linear non-homogeneous differential equations with variable coefficients in semi-infinite domain is

$$
\sum_{k=0}^{m} P_{k}(x) y^{(k)}(x)=f(x), \quad 0 \leq x<\infty
$$

with the mixed conditions

$$
\sum_{k=0}^{m-1} \sum_{j=0}^{J} d_{i j}^{k} y^{(k)}\left(b_{j}\right)=\alpha_{i}
$$

$$
0 \leq b_{j}<\infty, \quad i=0,1, \ldots, m-1, \quad j=0,1, \ldots, J
$$

where the $P_{k}(x)$ and $f(x)$ are continuous functions on the interval $[0, \infty), d_{i j}^{k}, b_{j}$ and $\alpha_{i}$ are appropriate constants or $b_{j}$ may tends to $\infty$.

Now, we consider that the approximate solution $y_{N}(x)$ to the exact solution $y(x)$ of Eq. (5) as

$$
y_{N}(x)=\sum_{n=0}^{N} a_{n} R_{n}(x)=R(x) A,
$$

and

$$
y_{N}^{(k)}(x) \cong \sum_{n=0}^{N} a_{n}\left(R_{n}(x)\right)^{(k)}=R^{(k)}(x) A,
$$

substituting the relation (4) into expression (8), we have the the scheme of the $(k)$ th-order derivative of the solution function $y_{N}(x)$ of the higher-order differential equations as

$$
\left[y_{N}^{(k)}(x)\right]=V^{(k)}(x) C^{T} A
$$

\section{Fundamental matrix relation based on collocation points}

Let us define the collocation points, so that $0 \leq x_{i} \leq \infty$, as 


$$
x_{i}=\frac{1+\cos \left(\frac{i \pi}{N}\right)}{1-\cos \left(\frac{i \pi}{N}\right)}, \quad i=1, \ldots, N-1
$$

and at the boundaries $(i=0, i=N) x_{0} \rightarrow \infty, x_{N}=0$, since the RC functions are convergent at one both boundaries $\infty$, the appearance of infinity in the collocation points does not cause a loss in the method.

Then, we substitute the collocation points (10) into Eq. (5) to obtain

$$
\sum_{k=0}^{m} P_{k}\left(x_{i}\right) y_{N}^{(k)}\left(x_{i}\right)=f\left(x_{i}\right), \quad i=0,1, \ldots, N .
$$

The system (11) can be written in the matrix form

$$
\sum_{k=0}^{m} \boldsymbol{P}_{k} \boldsymbol{Y}^{(k)}=\boldsymbol{F}
$$

where

$$
\boldsymbol{P}_{k}=\left[\begin{array}{cccc}
P_{k}\left(x_{0}\right) & 0 & \ldots & 0 \\
0 & P_{k}\left(x_{1}\right) & \ldots & 0 \\
0 & 0 & \ddots & \vdots \\
0 & 0 & \ldots & P_{k}\left(x_{N}\right)
\end{array}\right], \quad \boldsymbol{F}=\left[\begin{array}{llll}
f\left(x_{0}\right) & f\left(x_{1}\right) & \ldots & f\left(x_{N}\right)
\end{array}\right]^{T}
$$

By putting the collocation points $x_{i}$ in (9), we have the system

$$
\left[y_{N}^{(k)}\left(x_{i}\right)\right]=V^{(k)}\left(x_{i}\right) C^{T} A, \quad i=0,1, \ldots, N
$$

or

$$
\boldsymbol{Y}^{(k)}=\left[\begin{array}{c}
y_{N}^{(k)}\left(x_{0}\right) \\
y_{N}^{(k)}\left(x_{1}\right) \\
\vdots \\
y_{N}^{(k)}\left(x_{N}\right)
\end{array}\right]=\boldsymbol{V}^{(k)}(x) C^{T} A, \boldsymbol{V}^{(k)}=\left[\begin{array}{c}
V^{(k)}\left(x_{0}\right) \\
V^{(k)}\left(x_{1}\right) \\
\vdots \\
V^{(k)}\left(x_{N}\right)
\end{array}\right]=\left[\begin{array}{cccc}
v^{(0)}\left(x_{0}\right) & v^{(1)}\left(x_{0}\right) & \ldots & v^{(N)}\left(x_{0}\right) \\
v^{(0)}\left(x_{1}\right) & v^{(1)}\left(x_{1}\right) & \ldots & v^{(N)}\left(x_{1}\right) \\
\vdots & \vdots & \ddots & \vdots \\
v^{(0)}\left(x_{N}\right) & v^{(1)}\left(x_{N}\right) & \ldots & v^{(N)}\left(x_{N}\right)
\end{array}\right]
$$

And the fundamental matrix will be in the form

$$
\sum_{k=0}^{m} \boldsymbol{P}_{k} \boldsymbol{V}^{(k)}(x) C^{T} A=\boldsymbol{F}
$$

Next the corresponding matrix form for the condition can be written as follows

$$
\sum_{k=0}^{m-1} \sum_{j=0}^{J} d_{i j}^{k} \boldsymbol{V}^{(k)}\left(b_{j}\right) C^{T} A=\left[\alpha_{i}\right],
$$

$0 \leq b_{j}<\infty, \quad i=0,1, \ldots, m-1, \quad j=0,1, \ldots, J$

\section{5- Main Results}

The fundamental matrix Eq. (14) for Eq. (5) corresponds to a system of $(N+1)$ algebraic equations for the $(N+1)$ unknown coefficients $a_{0}, a_{1}, \ldots, a_{N}$.

One writes Eq. (14) in short form as:

$W A=\boldsymbol{F}$ or $[W ; \boldsymbol{F}]$

so that 


$$
W=\left[w_{p q}\right]=\sum_{k=0}^{m} \boldsymbol{P}_{k} \boldsymbol{V}^{(k)} C^{T} \quad p, q=0,1, \ldots, N
$$

We can obtain the matrix form for the mixed conditions (6), by means of Eq. (2), briefly, as

$$
U_{i} A=\alpha_{i} ; \quad \text { or } \quad\left[U_{i} ; \alpha_{i}\right] \quad i=0,1, \ldots, m-1,
$$

where

$$
U_{i}=\sum_{k=0}^{m-1} \sum_{j=0}^{J} d_{i j}^{k} \boldsymbol{V}^{(k)}\left(b_{j}\right) C^{T}
$$

Now, the solution of Eq. (5) under the conditions (6) can then be obtained by replacing the rows of matrices (17) by the any $m$ rows of the matrix (16), we get the required augmented matrix

$$
[\tilde{W} ; \tilde{\boldsymbol{F}}]=\left[\begin{array}{cccccc}
w_{00} & w_{01} & \ldots & w_{0 N} & ; & f\left(x_{0}\right) \\
w_{10} & w_{11} & \ldots & w_{1 N} & ; & f\left(x_{1}\right) \\
\ldots & \ldots & \ldots & \ldots & ; & \ldots \\
w_{N-m, 0} & w_{N-m, 1} & \ldots & w_{N-m, N} & ; & f\left(x_{N-m}\right) \\
u_{00} & u_{01} & \ldots & u_{0 N} & ; & \alpha_{0} \\
u_{10} & u_{11} & \ldots & u_{1 N} & ; & \alpha_{1} \\
\ldots & \ldots & \ldots & \ldots & ; & \ldots \\
u_{m-1,0} & u_{m-1,1} & \ldots & u_{m-1, N} & ; & \alpha_{m-1}
\end{array}\right]
$$

If $\operatorname{rank} \tilde{W}=\operatorname{rank}[\tilde{W} ; \tilde{\boldsymbol{F}}]=N+1$, then we can write the matrix equation (16) as:

$$
A=(\tilde{W})^{-1} \tilde{\boldsymbol{F}}
$$

and therefore the coefficients $a_{n} ; n=0,1, \ldots, N$ are uniquely determined by Eq.(18)

\section{6- Test Examples}

\section{Example 1}

Let us consider the following two point boundary value problem [9]

$$
y^{\prime \prime}(x)-\frac{1-x}{(1+x)^{2}} y(x)=\frac{1}{(x+1)^{2}}, \quad x \in[0, \infty)
$$

with $y(0)=1, y(x) \rightarrow 0$ when $x \rightarrow \infty$

For this example we have,

$m=2, \quad P_{0}(x)=\frac{x-1}{(x+1)^{2}}, \quad P_{1}(x)=0, \quad P_{2}(x)=1, \quad f(x)=\frac{1}{(x+1)^{2}}$.

Then, for $N=2$, the collocation points are

$$
x_{0} \rightarrow \infty, x_{1}=1, x_{2}=0 \text {, }
$$

and the fundamental matrix equation of problem is

$$
\left\{\boldsymbol{P}_{0} \boldsymbol{V}^{(0)} C^{T}+\boldsymbol{P}_{1} \boldsymbol{V}^{(1)} C^{T}+\boldsymbol{P}_{2} \boldsymbol{V}^{(2)} C^{T}\right\} A=\boldsymbol{F}
$$

Following the procedure in Section (5), we find the matrix in (18) for $N=2$ as: 


$$
[\tilde{W} ; \tilde{\boldsymbol{F}}]=\left[\begin{array}{ccccc}
1 & -1 & 1 & ; & 1 \\
1 & 1 & 1 & ; & 0 \\
-1 & -3 & 31 & ; & 1
\end{array}\right]
$$

we then obtain the $\mathrm{R} C$ coefficients as

$$
A=\left[\begin{array}{lll}
\frac{1}{2} & -\frac{1}{2} & 0
\end{array}\right]
$$

Therefore, we find the solution

$$
y(x)=\frac{1}{x+1}
$$

which is the exact solution of Example 1.

\section{Example 2}

Whittaker's equation eigenproblem [3]

$$
y^{\prime \prime}(x)+\left[\frac{-1}{4}+\frac{1}{y}+\frac{\lambda}{y}\right] y(x)=0, \quad x \in[0, \infty)
$$

where $\lambda$ is the eigenvalue is a special case of Whittaker's equation. The exact solution is $y=e^{-0.5 x} x L_{n}^{1}(y)$ where $\lambda=n, \quad n \geq 0 \quad$ is an integer where $L_{n}^{1}$ is the associated Laguerre polynomial of first order and degree $n$.

In special case $\lambda=-1$, Whittaker's equation take a form

$$
y^{\prime \prime}(x)-\frac{1}{4} y(x)=0, \quad x \in[0, \infty)
$$

the exact solution is $y(x)=e^{-0.5 x}$ with $y(0)=1, y(x) \rightarrow 0$ when $x \rightarrow \infty$.

We applied the RC collocation method and solved this problem. In Table 1, 2 the resulting values for $N=12,30$ and 50 using the present method together with the exact values of $y(x)=e^{-0.5 x}$ are tabulated. The error decreases when the integer $N$ is increased. The computing of the norms $L_{2}$ and $L_{\infty}$ in interval $x \in[0,4]$ given in Table 3 where

$$
L_{2}=\sqrt{h \sum_{i=0}^{l}\left(y_{\text {Exact }}^{i}-y_{\text {Appr }}^{i}\right)^{2}}, \quad L_{\infty}=\operatorname{Max}\left|\left(y_{\text {Exact }}^{i}-y_{\text {Appr }}^{i}\right)\right| .
$$

In addition the absolute errors for various $N$ are plotted in Fig.1.

Table 1 Comparison between Exact solution and approximate solutions obtained by present method for $y(x)$ of Example 2

\begin{tabular}{|c|c|c|c|c|}
\hline$x$ & Exact solution & $\begin{array}{c}\text { Present method } \\
N=12\end{array}$ & $\begin{array}{c}\text { Present method } \\
N=30\end{array}$ & $\begin{array}{c}\text { Present method } \\
N=50\end{array}$ \\
\hline 0 & 1 & 1 & 1 & 1 \\
\hline 0.2 & 0.904837418 & 0.904841460 & 0.904837421 & 0.904837355 \\
\hline 0.8 & 0.670320046 & 0.670350945 & 0.670320040 & 0.670319999 \\
\hline 1.2 & 0.548811636 & 0.548736621 & 0.548811594 & 0.548811597 \\
\hline 1.8 & 0.406569659 & 0.406632096 & 0.406569500 & 0.406569632 \\
\hline 2.2 & 0.332871083 & 0.333023241 & 0.332871381 & 0.332871061 \\
\hline 2.8 & 0.246596963 & 0.246624962 & 0.246596581 & 0.246596946 \\
\hline 3.2 & 0.201896517 & 0.201767625 & 0.201897064 & 0.201896505 \\
\hline 3.8 & 0.149568619 & 0.149258878 & 0.149568646 & 0.149568608 \\
\hline 4.2 & 0.122456428 & 0.122090995 & 0.122455511 & 0.122456423 \\
\hline 4.8 & 0.090717953 & 0.090341728 & 0.090717443 & 0.090717944 \\
\hline
\end{tabular}


Table 2 Comparison between absolute error functions obtained by present method for $y(x)$ of Example 2 for $\mathbf{N}=12,30$ and 50

\begin{tabular}{|c|c|c|c|}
\hline$x$ & $e_{12}$ & $e_{30}$ & $e_{50}$ \\
\hline 0 & 0 & 0 & 0 \\
\hline 0.2 & $4.04215 \mathrm{e}-006$ & $3.02671 \mathrm{e}-009$ & $6.25568 \mathrm{e}-008$ \\
\hline 0.8 & $3.08992 \mathrm{e}-005$ & $5.12635 \mathrm{e}-008$ & $4.60875 \mathrm{e}-008$ \\
\hline 1.2 & $7.50144 \mathrm{e}-005$ & $4.20386 \mathrm{e}-008$ & $3.85997 \mathrm{e}-008$ \\
\hline 1.8 & $6.24372 \mathrm{e}-005$ & $1.59395 \mathrm{e}-007$ & $2.71621 \mathrm{e}-008$ \\
\hline 2.2 & $1.52157 \mathrm{e}-004$ & $2.97549 \mathrm{e}-007$ & $2.24228 \mathrm{e}-008$ \\
\hline 2.8 & $2.79987 \mathrm{e}-005$ & $3.82429 \mathrm{e}-007$ & $1.74667 \mathrm{e}-008$ \\
\hline 3.2 & $1.28892 \mathrm{e}-004$ & $5.46065 \mathrm{e}-007$ & $1.25714 \mathrm{e}-008$ \\
\hline 3.8 & $3.09741 \mathrm{e}-004$ & $2.70375 \mathrm{e}-008$ & $8.62534 \mathrm{e}-009$ \\
\hline 4.2 & $3.65432 \mathrm{e}-004$ & $9.17214 \mathrm{e}-007$ & $5.30536 \mathrm{e}-009$ \\
\hline 4.8 & $3.76224 \mathrm{e}-004$ & $5.09952 \mathrm{e}-007$ & $8.83256 \mathrm{e}-009$ \\
\hline
\end{tabular}

Table 3 comparing the $L_{2}$ and $L_{\infty}$ in interval $x \in[0,4]$

\begin{tabular}{|l|l|l|}
\hline $\boldsymbol{N}$ & $L_{2}$ & $L_{\infty}$ \\
\hline 12 & $5.37104 \mathrm{e}-006$ & $3.43829 \mathrm{e}-004$ \\
\hline 30 & $1.66144 \mathrm{e}-011$ & $7.18677 \mathrm{e}-007$ \\
\hline 50 & $1.40474 \mathrm{e}-015$ & $6.58175 \mathrm{e}-008$ \\
\hline
\end{tabular}
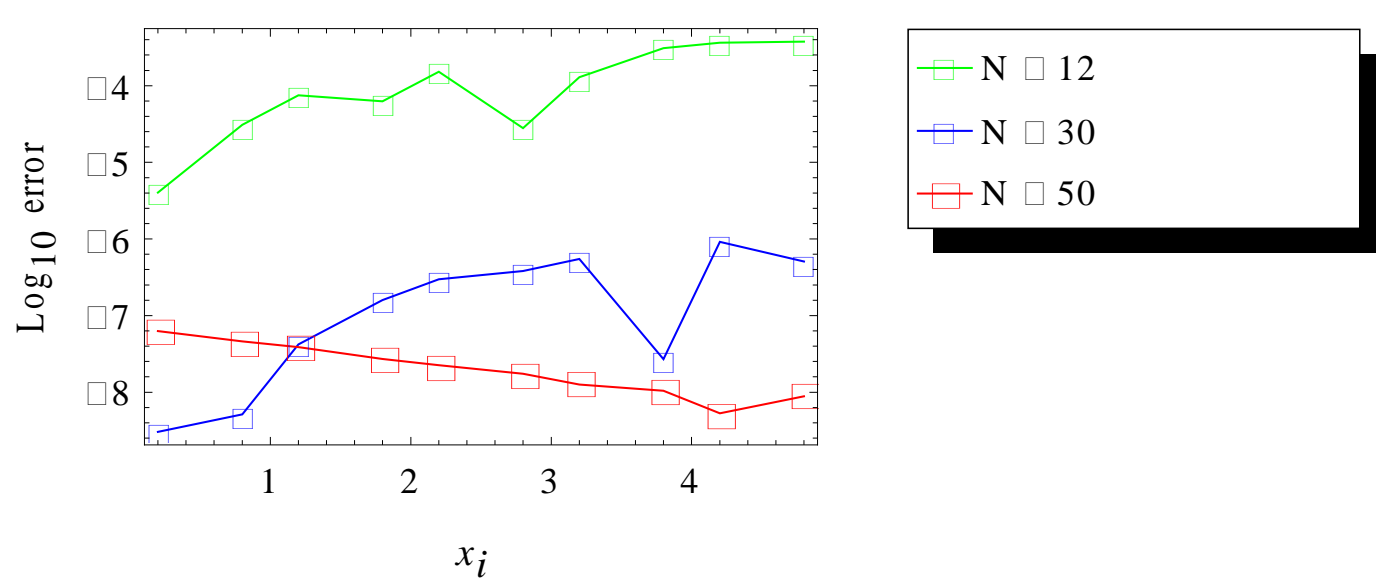

Fig. 1 the absolute errors for $N=12,30$ and 50

\section{Example 3}

Laguerre eigenproblem [3]

$$
x y^{\prime \prime}(x)+(x+1) y^{\prime}(x)+\lambda y(x)=0
$$

has the exact eigensolutions

$$
\begin{array}{llrl}
y(x) & =1, & \lambda & =0 \\
y(x) & =e^{-y} L_{n}(x), & \lambda & =n+1, \quad n \geq 0,
\end{array}
$$


where $L_{n}(x)$ are the usual Laguerre polynomials and the boundary conditions are

" natural" at both endpoints where $y(0)=1, y(x) \rightarrow 1$ when $x \rightarrow \infty$

Following the procedures in the previous examples, we obtain the solution for $N=2$ of this equation when $\lambda=0$, in the form $A=\left[\begin{array}{lll}1 & 0 & 0\end{array}\right]$.

Therefore, we find the solution $y(x)=1$, which is the exact eigensolution of Example 3 .

\section{7- Conclusion}

In this paper a rational Chebyshev $(\mathrm{RC})$ collocation method is investigated. The definition of the RC functions with new collocation points introduced to solve higher-order linear ordinary differential equations with variable coefficients in semiinfinite interval. The proposed differential equations and the given conditions were transformed to matrix equation with unknown RC coefficients. This technique is considered to be a modification of the similar presented in [7-10]. On the other hand, the RC functions approach deals directly with infinite boundary without singularities. This variant for our method gave us freedom to solve differential equations with boundary conditions tends to infinity. In addition, an interesting feature of this method is to find the analytical solutions if the equation has an exact solution that is a rational functions. Illustrative examples are used to demonstrate the applicability and the effectiveness of the proposed technique. The method can be extended for the case of systems of linear differential equations with variable coefficients which is under investigation by the authors.

\section{REFERENCES}

[1] J.C. Mason, D.C. Handscomb, Chebyshev polynomials, CRC Press, Boca Raton, 2003.

[2] L. Fox and I.B. Parker, Chebyshev polynomials in Numerical Analysis, Oxford University press, Ely House, London, 1968.

[3] J.P. Boyd, Orthogonal rational functions on a semi-infinite interval, J. Comput. Phys. 70, 63-88, 1987.

[4] J.P. Boyd, Chebyshev and Fourier spectral methods, Second Edition, DOVER Publications, Mineola, 2000.

[5] K. Parand and M. Razzaghi, Rational Chebyshev tau method for solving higher- order ordinary differential equations, Inter. J. Comput. Math. 81, 73-80, 2004.

[6] K. Parand and M. Razzaghi, Rational Chebyshev tau method for solving Volterra population model, Applied Mathematics and Computation 149, 893-900, 2004.

[7] M. Sezer, M.Gulsu and B. Tanay, Rational Chebyshev collocation method for solving higher-order linear ordinary differential equations, Wiley Online Library ,DOI 10.1002/num.20573, 2010.

[8] S. Yalcinbas, N.Ozsoy, M. Sezer, Approximat solution of higher-order linear differential equations by means of a new rational Chebyshev collocation method, Mathematical and Computational Applications, 15: 1, 45-56, 2010.

[9] M.A. Ramadan, K. R. Raslan, M. A. Nassar, An approximate analytical solution of higher-order linear differential equations with variable coefficients using improved rational Chebyshev collocation method, Applied and Computational Mathematics 3: 6, 315-322, 2014.

[10] M. A. Ramadan, K.R. Raslan, M. A. Nassar, An approximate solution of systems of high-order linear differential equations with variable coefficients by means of a rational Chebyshev collocation method, Electronic Journal of Mathematical Analysis and Applications, Vol. 4(1), Jan. (2016) pp. 86-98. 